Check for updates

Cite this: RSC Adv., 2017, 7, 33106

Received 3rd May 2017

Accepted 16th June 2017

DOI: $10.1039 / \mathrm{c} 7 \mathrm{ra0} 4989 \mathrm{~g}$

rsc.li/rsc-advances

\section{Mn doped CdS passivated CulnSe 2 quantum dot sensitized solar cells with remarkably enhanced photovoltaic efficiency}

\author{
Ikhtisham Mehmood, $\uparrow^{\mathrm{a}}$ Yueli Liu, $\uparrow^{\mathrm{a}}$ Keqiang Chen, ${ }^{\mathrm{a}}$ Abdul Hakim Shah ${ }^{\mathrm{b}}$ \\ and Wen Chen (D)*a
}

\begin{abstract}
Here we address the optoelectronics properties of $\mathrm{CulnSe}_{2}$ and $\mathrm{Mn}$ doped $\mathrm{CdS}$ quantum dot (CISe/Mn-CdS QD) passivated layer solar cells. CISe/Mn-CdS QD solar cells exhibit a remarkably enhanced optical absorption in the visible region as well as monochromatic incident photon-to-electron conversion efficiency (IPCE) (up to 40-90\%), which present the interesting photovoltaic efficiency of $3.96 \%$ under one sun illumination. Such remarkable optical behavior of the CISe/Mn-CdS QD passivated solar cells is explained by an optical mechanism based on an energy band model and valid physical laws.
\end{abstract}

\section{Introduction}

Quantum dot sensitized solar cells (QDSSCs) own many multidimensional characteristics, such as size tunable band gaps, ultrafast electron transfer, multi-exciton generation (MEG), high absorption and surpassing the Shockley-Queisser limit $(32 \%)$, which have attracted the profound attention of researchers over the last few decades. ${ }^{\mathbf{1 , 2}}$ Based on these exciting properties, Cd and Pb-based QDSSCs (CdS, CdSe, CdS/CdTe and $\mathrm{PbS}$, etc.) have been found to exhibit a power conversion efficiency (PCE) in the range of $4-9 \% .^{3-7}$ Zhong's group has obtained a PCE of $9.73 \%$ for a metal hydroxide coated CdSeTe photoanode. $^{8}$ Furthermore, an enhanced PCE of 11\% has been reported for the CdSeT QDSSCs with a carbon counter electrode. ${ }^{9}$ However, the toxic natures of $\mathrm{Cd}$ and $\mathrm{Pb}$ and the midgap trapping in $\mathrm{Pb}$ limit their commercial applications. ${ }^{\mathbf{1 0 , 1 1}}$

In order to solve these problems, researchers have shown their devotion towards the ternary semiconductor quantum dots (TQDs) in photovoltaic devices due to their non-toxic nature, narrow band gap, broad band light harvesting and low cost, such as $\mathrm{CuInS}_{2}, \mathrm{CuInSe}_{2}, \mathrm{AgInS}_{2}$, etc. ${ }^{\mathbf{1 2 - 1 4}}$ Among these materials, CuInSe ${ }_{2}$ quantum dots (CISe QDs) with the narrow band gap $(1.04 \mathrm{eV})$ have been found to effectively enhance the photovoltaic performance of the QDSSCs due to the large exciton Bohr's radius, which matches well with the solar spectrum. ${ }^{15-18}$ Till now, various techniques have been reported to

${ }^{a}$ State Key Laboratory of Advanced Technology for Materials Synthesis and Processing, School of Materials Science and Engineering, Wuhan University of Technology, Wuhan 430070, P. R. China. E-mail: chenw@whut.edu.cn; Fax:+86-27-87760129; Tel: +8627-87651107

${ }^{b}$ Department of Material Physics and Nanotechnology, Khushal Khan Khattak University, Karak 27200, Pakistan

$\dagger$ These authors contributed equally to this study and share first authorship. synthesize the CuInSe ${ }_{2}$ QDs for using in QDSSCs, ${ }^{19-21}$ which include the controllable solvothermal method by incorporating the use of diphenyl phosphine selenide as Se precursor ${ }^{17}$ and oleylamine as a solvent and capping ligand. ${ }^{20}$ Recently, Zhong's group have incorporated $\mathrm{Zn}$ in the CISe to obtain the absorption spectra at $1000 \mathrm{~nm}$ with PCE at the range of $9 \% .{ }^{22}$ Efficiency has been further enhanced to be $12 \%$ by nitrogen doped mesoporous carbon counter electrode in $\mathrm{Zn}-\mathrm{Cu}$-In-Se quantum dots sensitized solar cells. ${ }^{23}$ However, these QDSSCs still suffer from the issues of low efficiency and poor stability under drastic condition comparing with the conventional solar cells. Therefore, some new areas are required to obtain stable and efficient structure in QDSSCs. These solar cells still need to obtain the optimal structure and fulfill the demanding of the industrial applications.

In order to improve the optical and electrical properties of QDSSCs, various deposition approaches have been employed for the loading and passivation of QDs on the surface of $\mathrm{TiO}_{2}$ matrix. In situ chemical bath deposition (CBD), successive ionic layer adsorption and reaction (SILAR) method, ${ }^{24-26}$ and ex situ assembly linking technique ${ }^{4}$ are commonly applied techniques. The surface passivation of QDs by semiconductor materials with large band gap such as CdS and $\mathrm{ZnS}$ is carried out to enhance the stability of QDs surface and photovoltaic performance by the prevention of leakage current, which delivers a significant power conversion efficiency (PCE), ${ }^{20,27,28}$ and high open circuit voltage $\left(V_{\mathrm{oc}}\right) .{ }^{13}$ The advantage of the $\mathrm{Mn}^{2+}$ doping creates an additional electronic state in the CdS host material, which reduces electron-hole recombination and increases a short current density of the QDSSCs. Furthermore, the Mn has dorbital $\left({ }^{4} \mathrm{~T}_{1}-{ }^{6} \mathrm{~A}_{1}\right)$ spin and orbital forbidden states, which increase the life time of the excited electron. ${ }^{29}$ In addition, this type of the engineering structure controls the interfacial charge recombination and updates PCE of the cell. The midgap state 
originated from Mn doping induces the electron to be trapped, which screens them from the recombination with holes and oxidized polysulfide electrolyte. ${ }^{29}$ Moreover, the addition of the dopant materials elevates the PCE value upto $5.4-9.0 \%$ by changing the energy level in case of Mn doped CdS/CdSe and $\mathrm{ZnS} / \mathrm{SiO}_{2}$ QDSSCs. $^{27,29}$ Therefore, the engineering of Mn-doped material will greatly enhance the PCE of the QDSSCs.

Herein, we employ a low temperature and simple thermolysis method, followed by the capping of bifunctional molecule to prepare mercaptopropionic acid (MPA) capped CISe QDs, and the CISe QDs are sensitized on $\mathrm{TiO}_{2}$ matrix by in situ linker assisted technique. Furthermore, the passivation layer of Mndoped CdS (Mn-CdS) QDs over CISe QDs/ $\mathrm{TiO}_{2}$ matrix is carried out by SILAR method. ${ }^{27}$ The doping produces a stable structure under severe temperature condition and fast electron transfer. These CISe/Mn-CdS QD passivated QDSSCs exhibit significant optical and electrical properties under one sun illumination.

\section{Experimental}

\subsection{Synthesis of CuInSe $\mathrm{CDS}_{2}$}

All chemicals were analytically grade and were used without further purifications. CuInSe ${ }_{2}$ QDs were synthesized by a modified thermolysis method as reported in literature. ${ }^{18}$ Briefly, $10 \mathrm{~mL}$ oleylamine (OLA), $1 \mathrm{mmol}$ CuI $(0.190 \mathrm{~g})$ and $1 \mathrm{mmol} \mathrm{InCl}_{3}(0.220 \mathrm{~g})$ were simultaneously added to a three neck flask and degassed for 30 minutes. Then, the temperature was increased to $180{ }^{\circ} \mathrm{C}$ under the argon flow and $2 \mathrm{mmol} \mathrm{DPP} /$ Se (1: 1 ratio) was injected and the temperature was maintained at $180{ }^{\circ} \mathrm{C}$ for a further 10 minutes. The mixed solution was cooled to room temperature and washed by centrifugation through methanol and hexane for three times. The precipitates were dried at $100{ }^{\circ} \mathrm{C}$ in vacuum oven to obtain the CISe QDs powder.

The as-prepared CISe QDs were dispersed in fresh hexane for sensitization. To obtain MPA capped QDs, CISe QDs/methanol solution $(1 \mathrm{~mL})$ was dissolved in methanol solution of MPA and $\mathrm{NaOH}$, which was stirred for 30 minutes, then deionized water was added to the mixture and maintained stirring for further 20 minutes. The precipitates were finally washed through centrifugation in excess acetone and deionized water to obtain water soluble MPA capped CISe QDs.

\subsection{Device fabrication}

$\mathrm{TiO}_{2}$ nanoparticle working electrodes were prepared by screen printing technique as reported in literature. ${ }^{30}$ Briefly, fluorine doped tin oxide (FTO) glass was first treated with $\mathrm{TiCl}_{4}$ solution at $70{ }^{\circ} \mathrm{C}$ for 3 minutes. Back scattering layer was prepared from P25 paste, followed by the top layer of $400 \mathrm{~nm} \mathrm{TiO}_{2}$ nanoparticles. Finally, the $\mathrm{TiO}_{2}$ thin films were sintered at $450{ }^{\circ} \mathrm{C}$ in the muffle furnace, and then dipped in CISe QDs solution for 24 hours to complete the sensitization. These CISe QDs sensitized $\mathrm{TiO}_{2}$ films were washed by water and dried under vacuum.

MPA capped CISe QDs were sensitized on $\mathrm{TiO}_{2}$ films followed by the passivation of Mn-CdS layer by successive ionic layer adsorption and reaction (SILAR) method. In short, $0.1 \mathrm{M}$ cadmium nitrate in methanol was used as cation source and 0.1 M sodium sulfide in 1:1 methanol and water as anion source. To incorporate Mn doping, manganese acetate (0.075 M) was mixed with cadmium nitrate. The CISe QDs sensitized films were immersed for one minute in Mn doped Cd precursor followed by rainsing with methanol, and then immersed in $\mathrm{S}$ precursor followed by rinsing with methanol, which counts as one SILAR cycle and repeats the process for 3 cycles. Finally the devices were dried under Ar atmosphere to remove the organic solvents.

The QDs co-sensitized solar cells were fabricated by assembling the sensitized $\mathrm{TiO}_{2}$ photoanode and brass based $\mathrm{Cu}_{2} \mathrm{~S}$ counter electrode with a binder clip, separated by a $60 \mathrm{~mm}$ Scotch tape spacer. Polysulfide electrolyte solution with composition of $\mathrm{Na}_{2} \mathrm{~S}(2.0 \mathrm{M}), \mathrm{S}(2.0 \mathrm{M})$ powder and $\mathrm{KCl}(0.2 \mathrm{M})$ was injected into the cells device.

\subsection{Characterization and measurements}

Structure and morphology were measured by X-rays diffraction analysis (XRD, Pertpro, PANalytical, Netherlands) using $\mathrm{Cu} \mathrm{K} \alpha$, field emission scanning electron microscope (FESEM, JSEM$5610 \mathrm{LV}$, Japan) and the high resolution transmission electron microscope (HRTEM, JEM-2100F, JEOL, Japan), respectively. The optical characterization of the samples was carried out by using UV-vis absorption spectra (UV-2550, Shimadzu, Japan). The photocurrent density-voltage $(J-V)$ curves of the QDSSCs were measured by the Keithley 4200 semiconductor characterization system (Keithley Instruments, USA) under simulated AM 1.5 illumination (Newport 91160, $300 \mathrm{~W}$ xenon lamp, USA). The incident photon-to-current conversion efficiency (IPCE) was measured using Newport's IPCE Measurement Kit under the monochromatic light from a monochromator (Newport). The electrochemical impedance spectra (EIS) were measured by using an impedance analyzer (Auto lab PGSTAT320N) at opencircuit potentials under AM 1.5 illumination.

\section{Results and discussions}

XRD pattern of CISe QDs is shown in Fig. 1(a), and the diffraction peaks are indexed to the chalcopyrite (tetragonal) $\mathrm{CuInSe}_{2}$ QDs (JCPDS 00-051-1221) with all major peaks reflected from (112), (204), (312) and minor peak of (211) crystal plane at angle $2 \theta$ of $26.77^{\circ}, 44.43^{\circ}, 54.64^{\circ}$ and $31.01^{\circ}$, respectively, which is the stable phase of CISe QDs at room temperature. The significant broadening in the peaks represents the small size of $\mathrm{CuInSe}_{2}$ QDs. Applying Scherrer's formula, the average size of QDs is calculated to be $5 \mathrm{~nm} .{ }^{31}$ Fig. 1(b) and(c) illustrate the top and cross sectional views of the as-prepared film with the thickness of $6 \mu \mathrm{m}$. Average size of as-synthesized QDs is $5 \mathrm{~nm}$ as shown in the Fig. 1(d), which is in good agreement with the XRD analysis. The HRTEM image displays high crystallinity of the assynthesized CISe QDs in Fig. 1(e), and the inter-planner spacing is about $0.362 \mathrm{~nm}$, which corresponds to the (112) crystal plane of the tetragonal structure. 

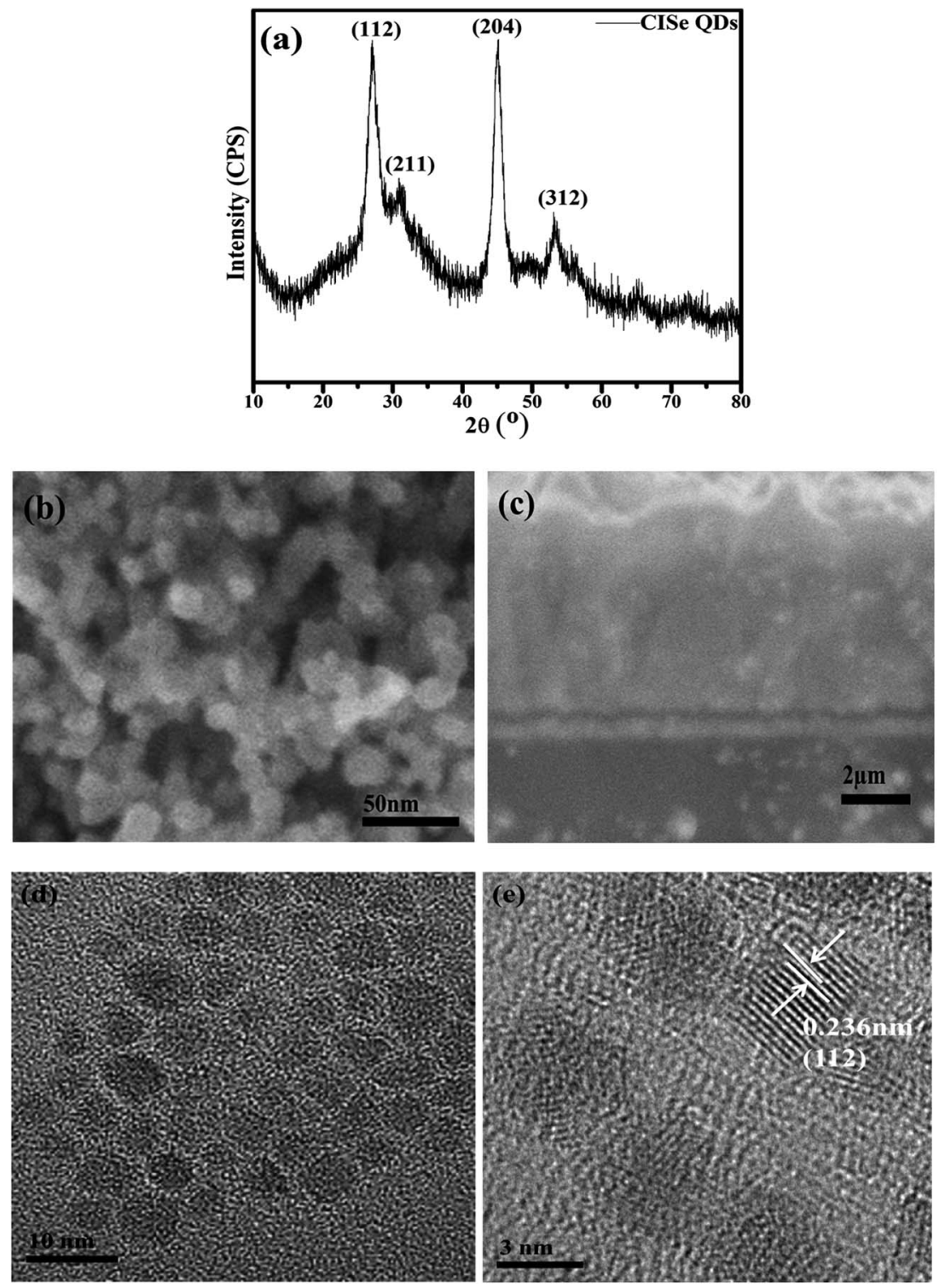

Fig. 1 Characteristic of the as-prepared CulnSe 2 quantum dots: (a) XRD pattern, (b) top and (c) cross-sectional views of FESEM images, (d) TEM image, (e) HRTEM image.

UV-vis spectra of the $\mathrm{TiO}_{2}$ photoanodes sensitized by CISe, CdS, Mn-CdS and CISe/Mn-CdS QDs are shown in Fig. 2(a). After the sensitization of CISe QDs, the absorption spectrum of the $\mathrm{TiO}_{2}$ films is significantly enhanced both in ultraviolet and visible light regions. The obvious blue shift is observed after the sensitization of CISe QDs with the main peak centered at $450 \mathrm{~nm}$, while the absorption onsets of the $\mathrm{TiO}_{2} / \mathrm{CdS}$ and $\mathrm{TiO}_{2} /$ Mn-CdS occur approximately at 560 and $580 \mathrm{~nm}$, respectively, which show the characteristics spectra toward red shift compared with that of the bulk CdS material. The CISe/Mn-CdS QD passivated electrode exhibits red-shift absorption at the wavelength range of 650-700 $\mathrm{nm}$. Mn-CdS passivation layer has an absorption onset at $580 \mathrm{~nm}$, the red-shift phenomenon must result from the light absorption of the CISe QDs. The passivation enhances the absorption spectra towards long wavelength region comparing with those of pure $\mathrm{TiO}_{2}$ and $\mathrm{CISe} / \mathrm{TiO}_{2}$ matrix.
This red-shift may indicate that the QDs charge carriers wave functions tunnel into the surrounding CdS shell. The broad spectrum reveals the heterogeneity of the CISe and Mn-CdS QDS sizes. ${ }^{32}$ Furthermore, UV-vis spectra are also measured to reveal the band gaps of the as-prepared samples. Herein, using Tauc relation (eqn (1)), the optical band gap of CISe QDs is calculated as $1.38 \mathrm{eV}$ and other corresponding materials are shown in Fig. 2(b). ${ }^{33,34}$

$$
\alpha h \nu=A\left(h \nu-E_{\mathrm{g}}\right)^{\frac{n}{2}}
$$

where $\alpha$ is absorption coefficient and given by $\alpha=2.303$, and $A$ $=\log (T / d)(d$ is the thickness of the sample and $T$ is the transmission), $h \nu$ is the photon energy. Further, the bands gaps of $\mathrm{TiO}_{2}, \mathrm{CdS}, \mathrm{Mn}-\mathrm{CdS}$ and CISe/Mn-CdS are 3.1, 2.3, 2.2 and $1.6 \mathrm{eV}$, respectively. 

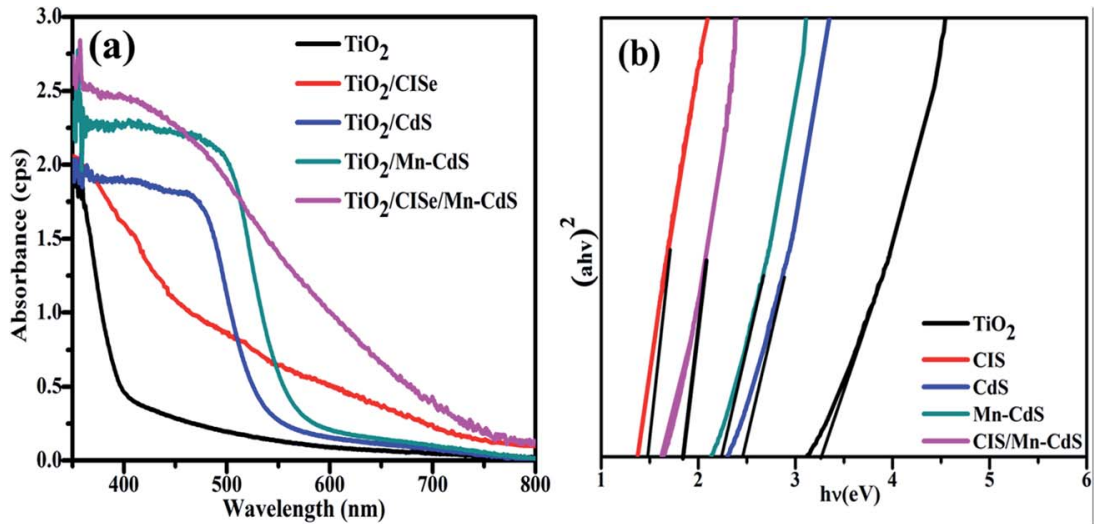

Fig. 2 (a) UV-vis spectra of $\mathrm{TiO}_{2}, \mathrm{CISe}, \mathrm{CdS}, \mathrm{Mn}-\mathrm{CdS}$ and $\mathrm{CISe} / \mathrm{Mn}-\mathrm{CdS}$ QDs sensitized solar cells, (b) the Tauc plots for the determination the band gap.
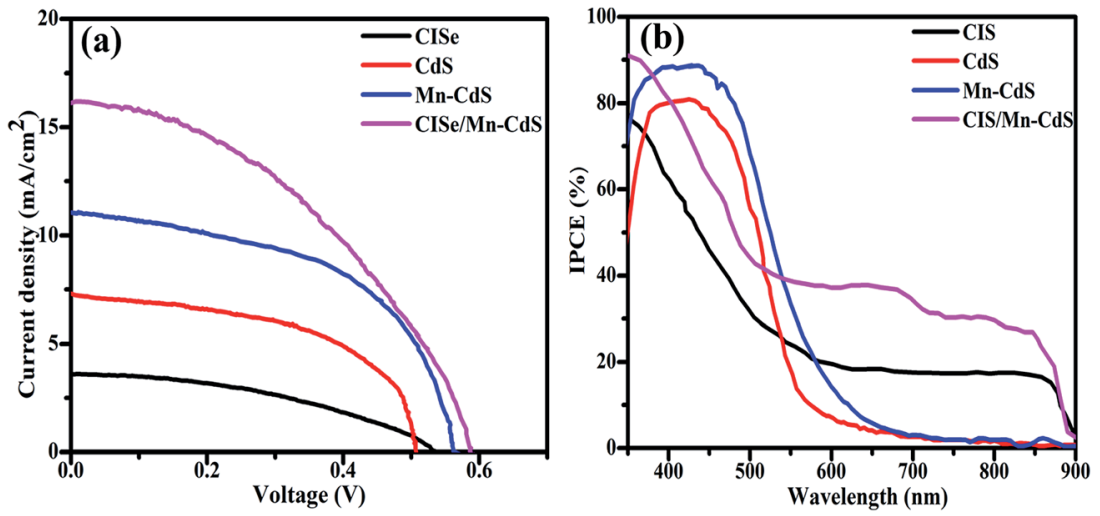

Fig. 3 The photovoltaic performance of the CISe, CdS, Mn-CdS and CISe/Mn-CdS sensitized solar cells under AM 1.5 sunlight: (a) J-V curves, (b) IPCE curves.

The photovoltaic performance is determined from the current-voltage $(J-V)$ characteristics of the assembled devices as shown in the Fig. 3(a). The photovoltaic parameters of these solar cells, such as the open circuit potential $\left(V_{\text {oc }}\right)$, short circuit current density $\left(J_{\mathrm{sc}}\right)$, fill factor $(\mathrm{FF})$ and power conversion efficiency (PCE), are listed in Table 1. The photovoltaic efficiencies of CISe QDs, CdS QDs, Mn-CdS QDs and CISe/Mn-CdS QD passivate solar cells are $0.80,1.98,3.08$ and 3.96\%, respectively. For CISe/Mn-CdS QD passivate solar cells, the decreasing of the $\mathrm{FF}$ to $41 \%$ clearly indicates the reduction in the internal charge recombination and defect state in the QDSSCs. ${ }^{18}$ Moreover, the increasing in the current density reveals the high charge transportation in photovoltaic device. CISe/Mn-CdS QD passivate solar cells exhibit an enhanced open circuit voltage of $0.59 \mathrm{~V}$, which substantiates a high charge extraction in the alloy structure. However, the obtained photovoltaic efficiency in the present work is still lower comparing with some of similar works. . $^{\mathbf{8} 18,22,29}$

Normally, the PCE value of QDSSCs is greatly influenced by the light absorption ability, which is related with the sensitization ratio of QDs as well as the size and morphology of the photoanode film. In our case, as the passivation of Mn-CdS QDs inside the pores of CISe QDs influences the electrolyte diffusion inside the photoanodes, which leads to lower the charge diffusion and ultimately limit the cell performance. ${ }^{35}$ The lower diffusion length causes the decreasing of the number of the extracted electrons to the $\mathrm{TiO}_{2}$ matrix. Conduction band level of CISe QDs is lower than that of n-type Mn-CdS QDs, which reduces the charge transfer across the CISe/Mn-CdS interface to cause a low photovoltaic performance. ${ }^{36}$ In our case, the SILAR and CBD deposition may lead to the undesired defect state due to the broad size distribution, thereby, the undesired surface defects are created, which leads to the trapping of charge carrier and the decreasing of the PCE value. ${ }^{29,37}$

IPCE is measured for electrons extraction/collection excited by incident photons as shown in Fig. 3(b). IPCE values of CISe, CdS, Mn-CdS and CISe/Mn-CdS QDSSCs show high values even in longer wavelength regions, and good consistency with the absorption spectra as shown in Fig. 2(a), which confirms the large number of electrons transport to the $\mathrm{TiO}_{2}$. The lower IPCE values of the CdS and Mn-CdS sensitized solar cells are due to their weak response at low energy wavelength. ${ }^{38}$ The enhancement in the IPCE value is noted for CISe/Mn-CdS QDSSCs with a maximum IPCE in the range of $40-90 \%$ with the obtained power conversion efficiency of $3.96 \%$. This high response of incident photon to excite electrons conversion is due to the 
Table 1 Photovoltaic parameters of various quantum dots sensitized solar cells extracted from $\mathrm{J}-\mathrm{V}$ measurements

\begin{tabular}{llcll}
\hline Type & $V_{\mathrm{oc}}(\mathrm{V})$ & $J_{\mathrm{sc}}\left(\mathrm{mA} \mathrm{cm}^{-2}\right)$ & $\mathrm{FF}$ & Efficiency (\%) \\
\hline CISe QD & 0.53 & 3.50 & 43 & 0.80 \\
CdS QDs & 0.51 & 7.20 & 52 & 1.98 \\
Mn-CdS QDs & 0.56 & 10.26 & 49 & 3.08 \\
CISe/Mn-CdS QD & 0.59 & 16.10 & 41 & 3.96
\end{tabular}

narrow band gap of CISe QDs, which can deduce a high electron injection upon the incident light photon. Moreover, the passivation of Mn-doped CdS QDs improves the performance of electron collection/injection, as the large number of the photoinduced electrons in CISe/Mn-CdS QDSSCs are injected into and then collected efficiently on $\mathrm{TiO}_{2}$ matrix. The high photoresponses in short and long wavelength regions are due to the combination of narrow and wide band gap CISe/Mn-CdS heterostructure, which absorbs the light in both of ultraviolet and visible regions. The photoresponse can be calculated by the following equation. ${ }^{39}$

$$
\mathrm{IPCE}=\mathrm{LHE} \times \phi_{\mathrm{inj}} \times \eta_{\mathrm{cc}}
$$

where LHE is the light harvesting efficiency, $\phi_{\text {inj }}$ is the electron injection efficiency and $\eta_{\mathrm{cc}}$ represents the charge collection efficiency ${ }^{39}$ Superior light harvesting and photoelectrochemical performance of CISe/Mn-CdS QDSSCs is another breakthrough for achieving the high photovoltaic efficiency.

QD loading ratio on the $\mathrm{TiO}_{2}$ surface is an important factor for high photovoltaic performance. The advantage of in situ chemicals bath deposition method is the insertion of the small size QDs in the space between $\mathrm{TiO}_{2}$ nanoparticles, thereby reduces the defect levels and electron-hole recombination, which ultimately enhances the photovoltaic performance. Firstly, MPA is a short chain ligand, which acts as the linker molecule between QDs and $\mathrm{TiO}_{2}$ thin film through a strong interaction of (Ti-O-Ti) with carboxyl group ( $\mathrm{COOH})$. Secondly, $\mathrm{SH}$ group of MPA firmly interacts with $\mathrm{Cu}$ ion through S-metal junction. ${ }^{4}$ Thirdly, according to soft and hard acid basis theory, $\mathrm{Se}^{2-}$ ions have a strong binding with soft ions-SH from MPA. ${ }^{40}$ The MPA capped and water soluble CISe QDs have a strong affinity to the $\mathrm{TiO}_{2}$ molecules. Moreover, under the simulated sunlight irradiation, the MPA linking effectively reduces the blocking of photo-induced electrons transfer and the recombination possibility of photo-induced electrons and holes, which results in the improvement of photovoltaic conversion efficiency. ${ }^{41}$

Electrical impedance spectra (EIS) expose the dynamics of bound or mobile charge in bulk or interfacial region inside the solar cells. The EIS measurements are carried out under the dark condition at different forward biases ranging from 0 to $-0.5 \mathrm{~V}$. Nyquist plots of the cells at forward biased $-0.5 \mathrm{~V}$ are shown in the Fig. 4. Comparing with the Nyquist plots of CISe, CdS and MnCdS QDSSCs, the CISe/Mn-CdS QDSSCs reveal a high $R_{\text {rec }}$ value, suggesting a considerably larger electron transport resistance between the photo-electrode and polysulfide electrolyte. ${ }^{32}$ The $R_{\mathrm{S}}$

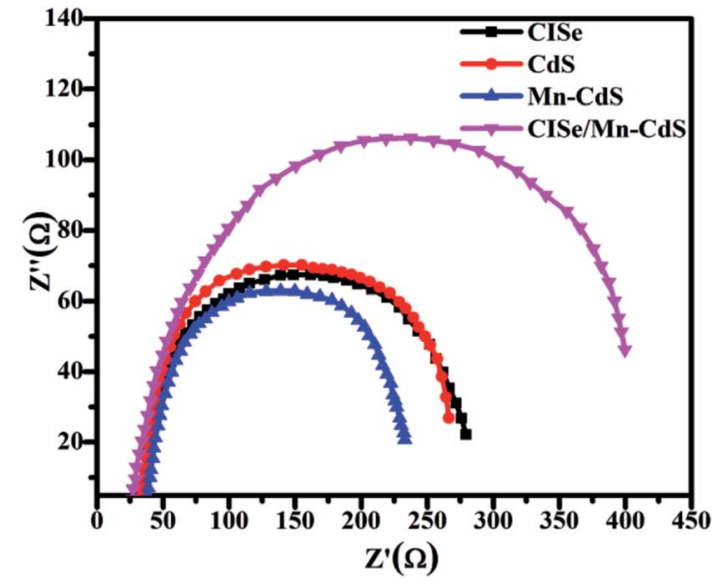

Fig. 4 EIS plots of CISe, CdS, Mn-CdS and CISe/Mn-CdS sensitized solar cells.

values of all the electrodes are nearly same, which is attributed to the fact that the passivation has no effect on the interfaces. Small semicircle of Mn-CdS QDSSCs indicates the favor of electron transfer from Mn-CdS QDs to the $\mathrm{TiO}_{2}$ matrix. The low resistance feature of the Mn-CdS QDs may be due to the forbidden dd orbital transition, which impulses the transportation electron and lowers the resistance. The large semicircle of CISe/Mn-CdS QDSSCs shows the high interface resistance, which is the sum of the resistances among $\mathrm{Mn}$-CdS QDs, CISe QDs and $\mathrm{TiO}_{2}$ interfaces in the QDSSCs and increases with the increasing of the film thickness. However, the surface defect and dangling bonds of the $\mathrm{TiO}_{2}$ film are removed by the Mn-CdS growth, which ultimately suppresses the photo-induced electron-hole recombination. In comparison with the other electron deterring approaches, the CISe/Mn-CdS alloy structure in our work exhibits a compelling performance in reducing the charge recombination and improving power conversion efficiency. The high recombination resistance of the Mn-CdS passivated CISe QDSSCs is possibly originated due to the surface trap states, which favor for the recombination to lower the PCE value of the device. The simulated results of the as-prepared samples are listed in the Table 2. These results confirm that the passivation of Mn-CdS QDs on CISe QDSSCs is an effective tool to suppress the charge recombination. It eventually contributes to the excellent properties of QDSSCs.

Under the sun light irradiation with sufficient energy, the photo-induced electrons jumps from valence band (VB) to conduction band (CB) of Mn-CdS QDs. Due to the large band gap offset, the photo-induced electrons transfer from the Mn-

Table 2 Simulated values of the resistance and capacitance of CISe, $\mathrm{CdS}, \mathrm{Mn}-\mathrm{CdS}$ and $\mathrm{CISe} / \mathrm{Mn}-\mathrm{CdS}$ QDSSCs

\begin{tabular}{llll}
\hline Sample & $R_{\mathrm{S}}\left(\Omega \mathrm{cm}^{2}\right)$ & $C_{\mu}\left(\mathrm{mF} \mathrm{cm}^{-2}\right)$ & $R_{\mathrm{rec}}\left(\Omega \mathrm{cm}^{2}\right)$ \\
\hline CISe QDSSCs & 30.54 & 0.51 & 285.9 \\
CdS QDSSCs & 30.20 & 0.46 & 271.4 \\
Mn-CdS QDSSCs & 36.30 & 0.54 & 223.8 \\
CISe/Mn-CdS QDSSCs & 24.80 & 0.72 & 443.0
\end{tabular}



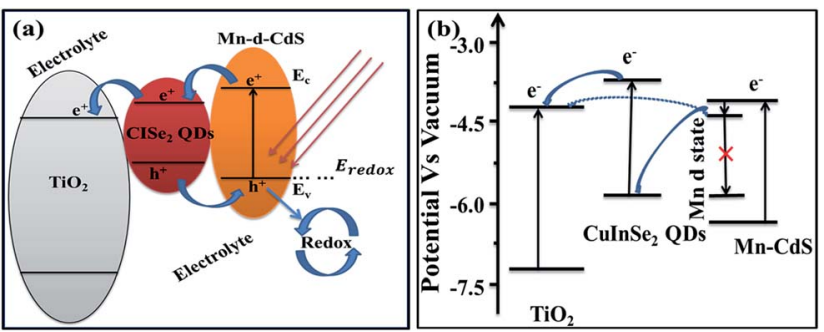

Fig. 5 Schematic diagrams of $\mathrm{ClSe} / \mathrm{Mn}-\mathrm{CdS}$ sensitized solar cells: (a) band gap, (b) electron transport path.

CdS CB to CuInSe 2 CB and then to $\mathrm{TiO}_{2} \mathrm{CB}$, which increases the photocurrent density. Furthermore, the photo-generated electrons from the CB of $\mathrm{CuInSe}_{2}$ QDs and Mn-CdS QDs with the high absorption of sun light transfer to $\mathrm{TiO}_{2} \mathrm{CB}$, which corporately increase the current density of the optoelectronic device. Hereafter, it is clearly observed that Mn doping causes the delay of the photo-induced electrons in the excited state, thereby enhances $V_{\text {oc }}$. Schematic diagram of the photo-induced electron flow is enlightened in Fig. 5. Mn doped CdS QDs create some states in the mid-bandgap region due to the $\mathrm{d}$ atomic orbitals of Mn $\left({ }^{4} \mathrm{~T} 1-{ }^{6} \mathrm{~A} 1\right)$. However, the transitions between these two energy levels $\left({ }^{4} \mathrm{~T} 1-{ }^{6} \mathrm{~A} 1\right)$ are both spin and orbital forbidden. ${ }^{27}$ Thereby, the photo-induced electrons stay for a long time at $\mathrm{Mn}$ d-states and then are injected to CISe QDs as well as to $\mathrm{TiO}_{2}$. There are some additional deep electron trap states, which reduce the concentration of mobile carriers in the n-type CdS layer. The generation of the excitons in the CdS QDs causes a compensation of deep trap by 'photo-doping, ${ }^{42,43}$ which increases the number of mobile carriers, hence reduces the barrier towards electron transport across the CdS QDs and leads to an increased junction conductance. ${ }^{44} \mathrm{Mn}-\mathrm{CdS}$ QDs have a higher mobility of photo-induced electrons than CdS QDs, which increases the current density of the Mn-CdS QDSSCs. Furthermore, the long life time of $\mathrm{d}-\mathrm{d}$ transition in Mn delays the electron-hole recombination in the QDs, which alternately increases the open circuit potential.

\section{Conclusion}

Water soluble CISe QDs of $5 \mathrm{~nm}$ are prepared by a simple thermolysis method. Co-sensitization of CISe/Mn-CdS QDs clearly enhances the optical and electrochemical properties of the solar cells. The excellent architecture of CISe/Mn-CdS demonstrates the outstanding light-harvesting behavior of the QDSSCs. MPA-capping improves the QDs loading on $\mathrm{TiO}_{2}$ photoanode. The combination of in situ linker and SILAR method in the fabrication of CISe/Mn-CdS alloy architecture suppresses the photo-induced electron-hole recombination; as a result, the photovoltaic efficiency of $3.96 \%$ is achieved for CISe/Mn-CdS QDSSCs.

\section{Acknowledgements}

This work is supported by the National Nature Science Foundation of China (No. 11674258, 51506155), International
Science \& Technology Cooperation Program of China (No. 2013DFR50710), the National Nature Science Foundation of Hubei Province (No. 2014CFB165, 2015CKC898), the Fundamental Research Funds for the Central Universities (No. 2017II22GX) and Applied Basic Research Program of Wuhan (No. 2016010101010020). Thanks for the measurements supporting from Center for Materials Research and Analysis at Wuhan University of Technology (WUT).

\section{References}

1 A. Kongkanand, K. Tvrdy, K. Takechi, M. Kuno and V. Prashant, J. Am. Chem. Soc., 2008, 130, 4007-4015.

2 C. J. Stolle, T. B. Harvey, D. R. Pernik, J. I. Hibbert, J. Du, D. J. Rhee, V. A. Akhavan, R. D. Schaller and B. A. Korgel, J. Phys. Chem. Lett., 2014, 5, 304-309.

3 X. Y. Yu, B. X. Lei, D. B. Kuang and C. Y. Su, J. Mater. Chem., 2012, 22, 12058-12063.

4 H. Zhang, K. Cheng, Y. M. Hou, Z. Fang, Z. X. Pan, W. J. Wu, J. L. Hua and X. H. Zhong, Chem. Commun., 2012, 48, 1123511237.

5 V. G. Pedro, C. Sima, G. Marzari, P. P. Boix, S. Gimenez, Q. Shen, T. Dittrich and I. M. Sero, Phys. Chem. Chem. Phys., 2013, 15, 13835-13843.

6 J. Yang, J. Wang, K. Zhao, T. Izuishi, Y. Li, Q. Shen and X. Zhong, J. Phys. Chem. C, 2015, 119, 28800-28808.

7 K. Zhao, Z. Pan, I. Mora-Sero, E. Canovas, H. Wang, Y. Song, X. Gong, J. Wang, M. Bonn, J. Bisquert and X. Zhong, J. Am. Chem. Soc., 2015, 137, 5602-5609.

8 Z. Du, Z. Pan, F. Santiago, K. Zhao, D. Long, H. Zhang, Y. Zhao, X. Zhong, J. Yu and J. Bisquert, J. Phys. Chem. Lett., 2016, 7, 3103-3111.

9 Z. Ren, Z. Wang, R. Wang, Z. Pan, X. Gong and X. Zhong, Chem. Mater., 2016, 28, 2323-2330.

10 J. Zhang, R. G. Du, Z. Q. Lin, Y. F. Zhu, Y. Guo, H. Q. Qi, L. Xu and C. J. Lin, Electrochim. Acta, 2012, 83, 59-64.

11 J. W. Lee, D. Y. Son, T. K. Ahn, H. W. Shin, I. Y. Kim, S. J. Hwang, M. J. Ko, S. Sul, H. Han and N. G. Park, Sci. Rep., 2013, 3, 1050.

12 T. Omata, K. Nose and O. Y. M. Shinya, J. Appl. Phys., 2009, 105, 073106.

13 D. V. Talapin, J. S. Lee, M. V. Kovalenko and E. V. Shevchenko, Chem. Rev., 2010, 110, 389-458.

14 B. Chen, H. Zhong and B. Zou, Prog. Chem., 2011, 23, 22762286.

15 Z. Pan, I. Sero, Q. Shen, H. Zhang, Y. Li, K. Zhao, J. Wang, X. Zhong and J. Bisquert, J. Am. Chem. Soc., 2014, 136, 9203-9210.

16 H. Zhong, Z. Wang, E. Bovero, Z. Lu, F. C. J. M. Van Veggel and G. D. Scholes, J. Phys. Chem. C, 2011, 115, 12396-12402.

17 M. G. Panthani, C. J. Stolle, D. K. Reid, D. J. Rhee, T. B. Harvey, V. A. Akhavan, Y. Yu and B. A. Korgel, J. Phys. Chem. Lett., 2013, 4, 2030-2034.

18 W. Li, Z. Pan and X. Zhong, J. Mater. Chem. A, 2015, 3, 16491655.

19 Q. Wang, J. Qiao, J. Zhou and S. Gao, Electrochim. Acta, 2015, 167, 470-475. 
20 C. F. Du, T. You, L. Jiang, S. Q. Yang, K. Zou, K. L. Han and W. Q. Deng, RSC Adv., 2014, 4(64), 33855-33860.

21 M. G. Panthani, V. Akhavan, B. Goodfellow, J. P. Schmidtke, L. Dunn, A. Dodabalapur, P. F. Barbara and B. A. Korgel, J. Am. Chem. Soc., 2008, 130, 16770-16777.

22 J. Du, Z. Du, J. S. Hu, Z. Pan, Q. Shen, J. Sun, D. Long, H. Dong, L. Sun, X. Zhong and L. J. Wan, J. Am. Chem. Soc., 2016, 138, 4201-4209.

23 S. Jiao, J. Du, Z. Du, D. Long, W. Jiang, Z. Pan, Y. Li and X. Zhong, J. Phys. Chem. Lett., 2017, 8, 559-564.

24 M. Booth, A. P. Brown, S. D. Evans and K. Critchley, Chem. Mater., 2012, 24, 2064-2070.

25 D. F. Watson, J. Phys. Chem. Lett., 2010, 1, 2299-2309.

26 D. R. Baker and P. V. Kamat, Adv. Funct. Mater., 2009, 19, 805-811.

27 P. K. Santra and P. V. Kamat, J. Am. Chem. Soc., 2012, 134, 2508-2511.

28 P. K. Santra and Y. S. Chen, Electrochim. Acta, 2014, 146, 654658.

29 J. Wang, Y. Li, Q. Shen, T. Izuishi, Z. Pan, K. Zhao and X. Zhong, J. Mater. Chem. A, 2016, 4, 877-886.

30 C. J. Barbé, F. Arendse, P. Comte, M. Jirousek, F. Lenzmann, V. Shklover and M. Grätzel, J. Am. Ceram. Soc., 1997, 80, 3157-3171.

31 A. Monshi, M. R. Foroughi and M. R. Monshi, World J. Nano Sci. Eng., 2012, 154-160.
32 S. B. Zhang, S. H. Wei and A. Zunger, J. Appl. Phys., 1998, 83, 31923196.

33 L. E. Brus, J. Chem. Phys., 1984, 80, 4403-4409.

34 A. S. Hassanien and A. A. Akl, J. Alloys Compd., 2015, 648, 280-290.

35 J. Luo, H. Wei, Q. Huang, X. Hu, H. Zhao, R. Yu, D. Li, Y. Luo and Q. Meng, Chem. Commun., 2013, 49, 3881-3883.

36 A. Niemegeers, M. Burgelman and A. D. Vos, Appl. Phys. Lett., 1995, 6, 843-845.

37 Q. Guo, S. J. Kim, M. Kar, W. N. Shafarman, R. W. Birkmire, E. A. Stach, R. Agrawal and H. W. Hillhouse, Nano Lett., 2008, 8, 2982-2987.

38 T. L. Li, Y. L. Lee and H. Teng, Energy Environ. Sci., 2012, 5, 5315-5324.

39 Z. Yang, C. Y. Chen, P. Roy and H. T. Chang, Chem. Commun., 2011, 47, 9561-9571.

40 R. G. Pearson, J. Am. Chem. Soc., 1963, 85, 3533-3539.

41 K. S. Leschkies, R. Divakar, J. Basu, E. Enachepommer, J. E. Boercker, C. B. Carter, U. R. Kortshagen, D. J. Norris and E. S. Aydil, Nano Lett., 2007, 7, 17931789.

42 I. M. Seró, S. Giménez, T. Moehl, F. F. Santiago, T. L. Villareal, R. Gómez and J. Bisquert, Nanotechnology, 2008, 19, 424007.

43 N. Alex, B. Marc and D. V. Alexis, Appl. Phys. Lett., 1995, 67, 843-845.

44 A. O. Pudov, J. R. Sites, M. A. Contreras, T. Nakada and H. W. Schock, Thin Solid Films, 2005, 273, 480-481. 